\title{
Ultrafiltration of Lipoproteins through a Synthetic Membrane
}

\author{
IMPLICATIONS FOR THE FILTRATION THEORY \\ OF ATHEROGENESIS
}

\author{
Clark K. Colton, Sigmund Friedman, Dana E. Wilson, and \\ ROBERT S. LeES \\ From the Clinical Research Center and Departments of Chemical Engineering \\ and Nutrition and Food Science, Massachusetts Institute of Technology, \\ Cambridge, Massachusetts 02142
}

A B S T RACT To investigate the interaction of lipoproteins with semipermeable membranes, solutions of low density lipoproteins (LDL), very low density lipoproteins (VLDL), mixtures of the two, and diluted, normal, and hyperlipidemic serum were ultrafiltered through a synthetic membrane (500 A nominal pore diameter) using a stirred laboratory ultrafiltration cell. The pressure dependence of ultrafiltrate flux showed that a concentrated layer of lipoproteins was built up at the membrane surface (concentration polarization) and that VLDL was more subject to polarization than LDL. This phenomenon controlled the observed lipoprotein transport behavior. Whereas true membrane rejection (the fraction of the solute on the membrane surface which does not pass through the membrane) was greater than 0.95 for both LDL and VLDL, observed solute rejection varied from nearly 0 to 1.0 , depending upon experimental conditions.

If concentration polarization occurs in the arterial system, these results suggest that lipoprotein transport into arterial wall may be influenced not only by arterial blood pressure and the properties of the arterial wall, but also by local hemodynamic conditions and by the relative as well as absolute magnitudes of LDL and VLDL concentration.

This study was presented in part before the 43rd Annual Scientific Sessions of the American Heart Association in Atlantic City, N. J., and published in abstract form (Circulation. 1970. 42 [Suppl. 3] : 8.)

$\mathrm{Mr}$. Friedman is presently at the State University of New York College of Medicine at New York City. Dr. Wilson is presently at the College of Medicine, University of Utah, Salt Lake City, Utah.

Received for publication 13 July 1971 and in revised form 12 April 1972.

\section{INTRODUCTION}

In the human body, water passes continuously from the bloodstream across the walls of the blood vessels to the regional lymphatics as a result of a hydrostatic pressure difference, carrying with it some of the plasma solutes (1). The filtration theory of atherogenesis (2-9) hypothesizes that the earliest manifestations of atherosclerosis are intimately associated with this process; and, specifically, that the plasma lipoproteins, particularly the low density lipoproteins (LDL), ${ }^{1}$ are transported in this way into the arterial wall where some are trapped and deposit their lipid, eventually leading to the formation of atheroma.

Although the concept that lipids present in atherosclerotic lesions originate primarily from the blood plasma is quite old $(10,11)$, heretofore the filtration theory has been subjected to limited quantitative investigation. Courtice and Garlick (12) studied the transfer of plasma lipoproteins across the capillary walls to the

\footnotetext{
${ }^{1}$ Abbreviations used in this paper: LDL, low density lipoproteins; VLDL, very low density lipoproteins; A, membrane area available for transport $\left(\mathrm{cm}^{2}\right) ; b$, membrane radius for a circular model membrane $(\mathrm{cm}), \mathrm{c}_{\mathrm{b}}$, solute concentration in the bulk solution (retentate) $\left(\mathrm{mg} / \mathrm{cm}^{3}\right) ; \mathrm{c}_{\mathrm{f}}$, solute concentration in the ultrafiltrate $\left(\mathrm{mg} / \mathrm{cm}^{3}\right) ; c_{\mathrm{g}}$, maximum attainable solute concentration at the wall (membrane surface) $\left(\mathrm{mg} / \mathrm{cm}^{3}\right) ; c_{w}$, solute concentration at the wall (membrane surface) $\left(\mathrm{mg} / \mathrm{cm}^{3}\right) ; \exp (\mathrm{x}), \mathrm{e}^{\mathrm{x}} ; \mathrm{D}$, solute molecular diffusion coefficient $\left(\mathrm{cm}^{2} / \mathrm{sec}\right) ; J_{f}$, ultrafiltrate flux $\left(\mathrm{cm}^{3} /\left[\mathrm{min} \cdot \mathrm{cm}^{2}\right]\right)$; $\mathrm{J}_{\mathrm{s}}$, solute flux $\left(\mathrm{mg} /\left[\mathrm{min} \cdot \mathrm{cm}^{2}\right]\right) ; \mathrm{k}$, mass transfer coefficient $(\mathrm{cm} / \mathrm{min}) ; Q_{f}$, ultrafiltrate flow rate $\left(\mathrm{cm}^{3} / \mathrm{min}\right) ; R$, observed rejection of solute by membrane $\left(1-c_{f} / c_{b}\right) ; r$, true rejection of solute by membrane $\left(1-c_{f} / c_{w}\right) ; \delta$, hypothetical stagnant film thickness $(\mathrm{cm}) ; \nu$, kinematic viscosity $\left(\mathrm{cm}^{2} / \mathrm{sec}\right)$; $\omega$, impeller angular velocity in the ultrafiltration cell (radians/sec).
} 
lymph in the leg of hypercholesterolemic rabbits. The ultrafiltration of human serum through various tissues and the deposition of lipid therein has been investigated in vitro with excised human arteries and veins (13-15), human aortas (3), and aortas from various animals $(16,17)$. In vivo accumulation of labeled cholesterol in various layers of the aortic wall has been studied in the rat (4) and dog (18-20). The large amount of research dealing with lipid influx, deposition, and exchange in the arterial walls of laboratory animals has recently been reviewed (21), and various mechanisms to account for lipid accumulation and atheroma development have been proposed $(4,15,21-24)$.

Most of this work has focused on the influx or accumulation of specific lipids, such as cholesterol or phospholipid. Relatively little is known about the rates of influx and efflux of specific plasma lipoproteins in the arterial wall and the physiochemical phenomena on which their passage depends. As a preliminary approach to the investigation of these phenomena, we have studied the transport of LDL and very low density lipoproteins (VLDL) across a synthetic model membrane in a laboratory ultrafiltration system from solutions of single lipoproteins, mixtures, and serum. Ultrafiltration rates and lipoprotein transport were measured as a function of applied hydrostatic pressure and lipoprotein concentration.

\section{METHODS}

Experimental. The filtration studies were performed with lipoproteins from normal blood donors, using acid citrate dextrose (ACD) blood plasma supplied by the Massachusetts Red Cross through the kindness of Dr. Allen Kliman. The blood was drawn with the patients fasting; samples with detectable chylomicrons were not used. Serum was obtained when needed from blood drawn from volunteers who had fasted overnight; it was used within $24 \mathrm{hr}$.

VLDL and LDL were isolated by dialysis of the ACD plasma against solutions of $\mathrm{d} 1.006$ and 1.063 , respectively, followed by ultracentrifugation by techniques described elsewhere (25). The LDL was washed once by repeat ultracentrifugation. The VLDL was not washed in order to avoid aggregation.

The over-all experimental procedure is illustrated in Fig. 1. Solutions to be ultrafiltered were made up from the purified lipoprotein fractions or fresh whole serum and were diluted as desired with isotonic saline solution which was made $0.001 \mathrm{M}$ in disodium ethylenediaminetetraacetate (EDTA) and adjusted to $\mathrm{pH} 8.0$ with aqueous $\mathrm{NH}_{4} \mathrm{OH}$. After ultrafiltration through a synthetic nitrocellulose membrane (Sartorius, S-11310, 500 A nominal pore diameter), the LDL and VLDL in the ultrafiltrate and in the retentate were separated by a combination of ultracentrifugation and dextran-sulfate precipitation and quantitated in terms of their cholesterol content (25).

The membrane was contained at the bottom of a stirred, laboratory ultrafiltration cell (Amicon Corp., Lexington, Mass., model 52). The cell was filled to the top with the solution to be ultrafiltered, and fluid volume in the cell was

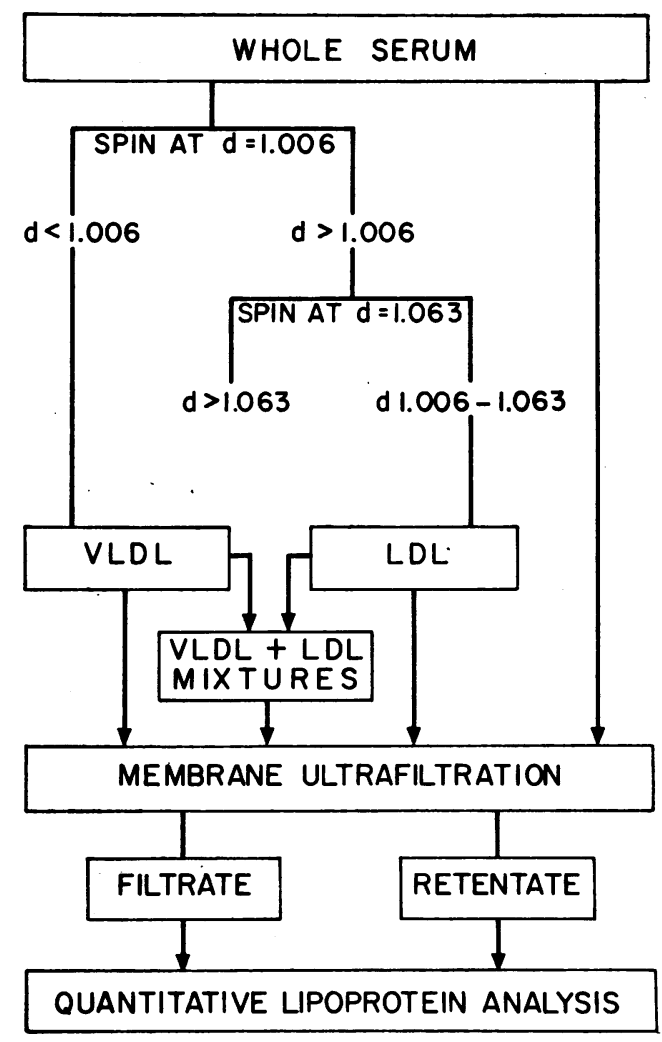

FIGURE 1 Schematic diagram of the experimental procedure. See text for details.

held constant by the addition of EDTA-saline solution from a reservoir maintained at the desired pressure by compressed nitrogen. This procedure prevented entrainment of air bubbles by the impeller. Applied pressure was measured with a pressure gauge and was varied from about $0.1-30 \mathrm{lb} . /$ inch $^{2}$ (about $5-1500 \mathrm{~mm} \mathrm{Hg}$ ). This circumscribes the physiological and pathological range of interest, roughly 1-6 lb./inch'. For pressures less than $3 \mathrm{lb}$./ inch $^{2}$, the reservoir tank was replaced by an elevated chamber filled with buffer. All experiments were carried out at $25 \pm 1^{\circ} \mathrm{C}$. Ultrafiltration flow rate was measured by timed collection into a graduated cylinder. About $12 \mathrm{ml}$ were collected for each sample; the first $2 \mathrm{ml}$ were discarded. The duration of each run depended upon the ultrafiltration rate and lasted from several minutes to several hours. During the course of a run, retentate concentration dropped slowly because of dilution. The variation was usually less than $10 \%$, and the average value is reported here. The ultrafiltration cell was agitated by a suspended magnetic stirrer bar run at $1200 \mathrm{rpm}$ as measured by a stroboscopic light source.

Measurement of LDL and VLDL triglyceride and protein content was carried out for selected runs. In all cases, the relative amounts of each component (cholesterol, triglyceride, and protein) in each lipoprotein class were the same in the ultrafiltrate and retentate. Furthermore, the LDL and VLDL in the ultrafiltrate were identical on double immunodiffusion (25) with LDL and VLDL in the retentate. All the lipoproteins introduced into the system could be accounted for in the ultrafiltrate and retentate within the 
range of experimental error. Hence, the amount of lipoprotein trapped within or on the membrane was negligibly small.

Calculations (see footnote 1 for definition of terms). The results are reported in terms of the ultrafiltrate flux

$$
J_{f}=\frac{Q_{f}}{A}
$$

and the observed solute rejection

$$
\mathrm{R}=1-\frac{\mathrm{c}_{\mathrm{f}}}{\mathrm{c}_{\mathrm{b}}}
$$

The solute (lipoprotein) flux, $\mathrm{J}_{\mathrm{s}}$, is calculated from

$$
\mathrm{J}_{\mathrm{s}}=\mathrm{J}_{\mathrm{f}} \mathrm{c}_{\mathrm{f}}=\mathrm{J}_{\mathrm{f}}(1-\mathrm{R}) \mathrm{c}_{\mathrm{b}}
$$

At fixed operating conditions, ultrafiltration flux and rejection showed no change with time or when fresh solutions were introduced. When exposure to pressures above $5 \mathrm{lb}$./inch ${ }^{2}$ was followed by a decrease in pressure, ultrafiltrate flux decreased and rejection increased slightly from their previous values. Consequently, all experiments were run in the order of increasing pressure to eliminate this modest hysteresis effect. A new membrane was used for each run, and all lipoprotein solutions (stored at $4^{\circ} \mathrm{C}$ ) were used within 3 days of preparation. Ultrafiltrate flux of pure buffer was measured for each new membrane, and those showing marked deviations from normal were discarded.

The transport rates of solutes and solvent during the ultrafiltration of solutions of macromolecules are often limited by mass transfer in the fluid phase adjacent to the membrane (26-30). If a membrane exhibits complete or partial rejection of a solute, i.e., failure to allow its free passage, solute will accumulate near the membrane surface. At steady state, the rate at which solute is brought to the membrane by fluid flow normal to it is balanced by solute transport through the membrane and by diffusion back into solution. The phenomenon of solute build-up at the membrane surface is generally referred to as concentration polarization. Combination of a mass balance over a differential volume element near the membrane, neglecting transport parallel to the membrane, with the simple film theory model of mass transfer (31) yields

$$
J_{f}=k \ln \left[\frac{c_{w}-c_{f}}{c_{b}-c_{f}}\right]
$$

For a nonzero convective velocity normal to the membrane, $c_{w}$ is always greater than $c_{b}$. Hence, one must distinguish between the experimentally observed rejection, $R$, and the true rejection intrinsic to the membrane.

$$
r=1-\frac{c_{f}}{c_{w}}
$$

The increased solute concentration at the membrane surface causes $R$ to be lower than $r$. The intrinsic membrane rejection itself is not a constant but increases with increasing ultrafiltration velocity (32).

Combination of equations 2,4 , and 5 , yields a number of useful relationships (31):

$$
\begin{aligned}
J_{f}=k \ln \left[\frac{\frac{c_{w}}{c_{b}}+R-1}{R}\right] \\
=k \ln \left[\frac{r\left(\frac{c_{w}}{c_{b}}\right)}{1-(1-r)\left(\frac{c_{w}}{c_{b}}\right)}\right]
\end{aligned}
$$

$$
\frac{c_{w}}{c_{b}}=R\left[\exp \left(J_{f} / k\right)-1\right]+1
$$

$$
=\frac{\exp \left(J_{f} / k\right)}{r+(1-r) \exp \left(J_{f} / k\right)}
$$

$$
r=\frac{R \exp \left(J_{f} / k\right)}{(1-R)+R \exp \left(J_{f} / k\right)}
$$

$$
R=\frac{r}{(1-r) \exp \left(J_{f} / k\right)+r}
$$

The observed rejection is dependent upon the true rejection and the dimensionless ratio $\mathrm{J}_{\ell} / \mathrm{k}$. The latter quantity is a measure of the relative importance of mass transferred by convection to the membrane surface as compared with mass transferred by diffusion away from the membrane surface. As $J_{t} / k$ becomes large, exp $\left(J_{t} / k\right)$ approaches infinity,

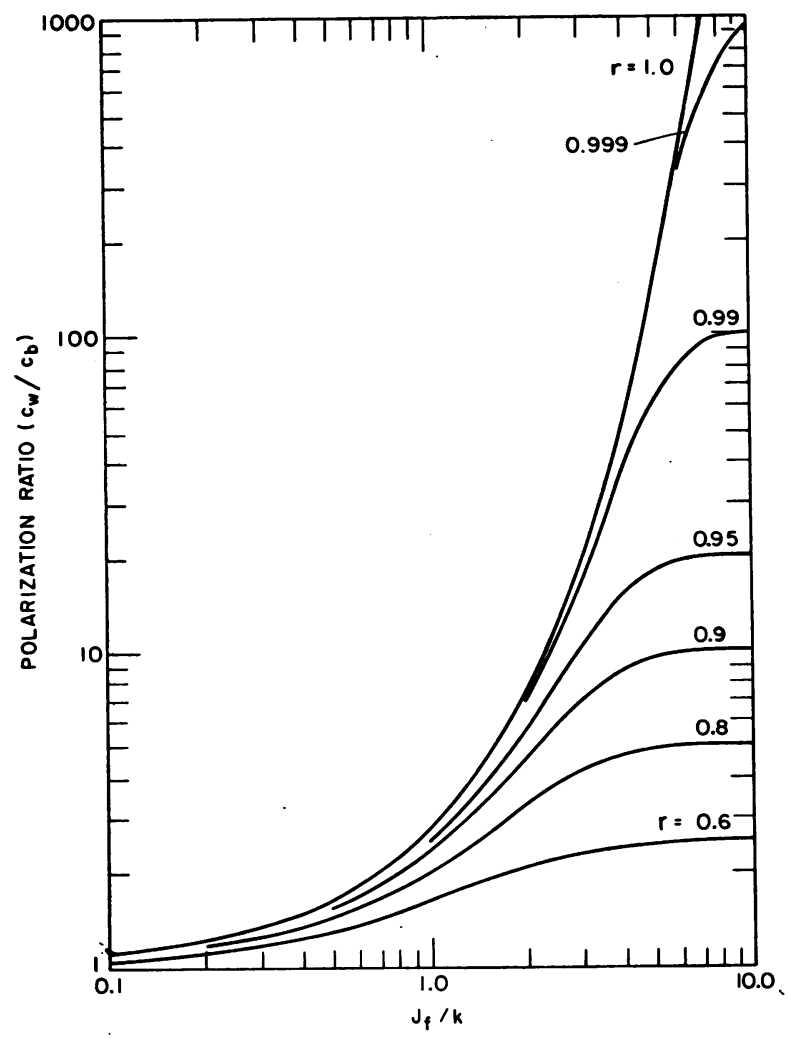

Figure 2 Dependence of polarization ratio, $c_{w} / c_{b}$, upon $J_{q} / k$ and true membrane rejection, $r$. Plotted curves are from equation 7. See footnote 1 for definition of terms. 


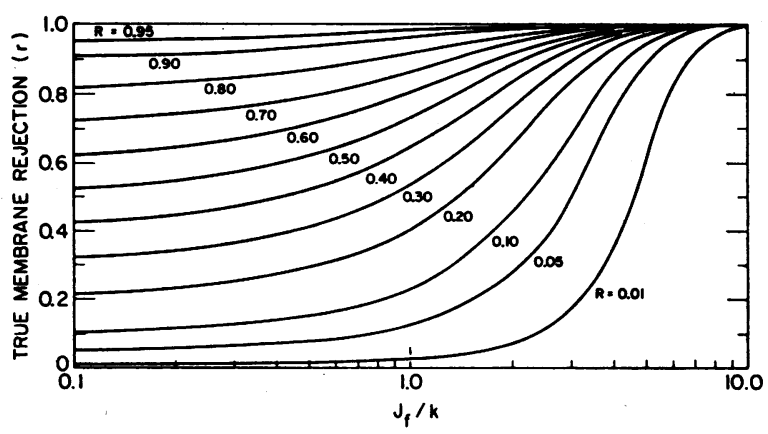

FIGURE 3 Effect of concentration polarization on observed solute rejection. See footnote 1 for definition of terms.

then $c_{w} / c_{b}$ approaches $1 /(1-r)$, and $R$ approaches zero. As $\mathrm{J}_{t} / \mathrm{k}$ tends to zero, exp $\left(\mathrm{J}_{\mathrm{f}} / \mathrm{k}\right)$ approaches one, then $\mathrm{c}_{w} / \mathrm{c}_{\mathrm{b}}$ approaches one, and $R$ approaches $r$.

Equation 7 is illustrated in Fig. 2, where the polarization ratio, $c_{w} / c_{b}$ is plotted as a function of $J_{t} / k$ for various values of $r$. The curves show the large values of $c_{w} / c_{b}$ which may be attained if the true membrane rejection is near unity. Equation 8 is plotted in Fig. 3 for a wide range of observed membrane rejections. The large influence that concentration polarization may have upon the measured rejection is evident. For example, at $\mathrm{J} / \mathrm{k}=5$, an observed rejection of 0.05 corresponds to a true membrane rejection of about 0.89 .

In real systems, $c_{w}$ must approach a maximum value which is dependent upon solubility limits, steric constraints in the close packing of molecules, or electrostatic repulsion effects. It is usually assumed $(29,30)$ that this results in the formation of a gel layer at the membrane surface characterized by a constant concentration, $c_{g}$. Estimates of $c_{z}$ from experimental data for different systems (30) vary from several weight per cent to nearly 100 weight $\%$, and a priori estimation is not possible.

When pure water is ultrafiltered, ultrafiltrate flux varies linearly with applied pressure. With macromolecular solutions, the flux is always lower and approaches a constant value at high pressure. The existence of such a maximum, pressure-independent flux is consistent with attainment of a constant solute concentration at the membrane surface. If $c_{w}$ (a variable) in Equation 6 is replaced by $c_{g}$ (a constant), $\mathrm{J}_{\mathrm{f}}$ is a function of $\mathrm{k}, \mathrm{r}, \mathrm{c}_{\mathrm{g}}$, and $\mathrm{c}_{\mathrm{b}}$, but no longer of applied pressure. The polarized layer thus behaves like a second, dynamically formed membrane with a hydrodynamic resistance to flow. It responds to an increase in pressure by thickening and/or changing its flow resistance characteristics.

To account for concentration polarization in data analysis, the mass transfer coefficient must be estimated. In this study the Reynolds number ${ }^{2}$ based on membrane radius $\left(\omega \mathrm{b}^{2} / \nu\right)$ is sufficiently high (about 56,000 for water at $25^{\circ} \mathrm{C}$ ) that the fluid boundary layer on the membrane is turbulent. In this case the radial variation in mass transfer coefficient is small (33), and the average value may be estimated with little error from the correlation of Smith, Colton, Merrill, and Evans (34). For the geometrical configuration of the

\footnotetext{
${ }^{2}$ A dimensionless number which characterizes the conditions of fluid flow.
}

Amicon model 52 cell

$$
k=1.94\left(\frac{D}{b}\right)\left(\frac{\nu}{D}\right)^{1 / 3}\left(\frac{\omega b^{2}}{\nu}\right)^{0.746}
$$

Using the viscosity of water at $25^{\circ} \mathrm{C}$ and a stirrer speed of $1200 \mathrm{rpm}$, equation 10 becomes

$$
\mathrm{k}=612 \mathrm{D}^{2 / 3}
$$

LDL are spherical particles with diameters ranging from 175 to 225 A $(12,25,36-38)$. The Stokes-Einstein equation (35) with an average LDL diameter of $190 \mathrm{~A}$ (25) gives a diffusion coefficient of $2.6 \times 10^{-7} \mathrm{~cm}^{2} / \mathrm{sec}$ in water at $25^{\circ} \mathrm{C}$ and, from equation 11, a mass transfer coefficient of 2.5 $\times 10^{-\mathbf{a}} \mathrm{cm} / \mathrm{min}$. VLDL are more polydisperse and probably represent a continuous overlapping of two or more related species (36) with diameters ranging from about 200 to $800 \mathrm{~A}$. An average value of $500 \mathrm{~A}$ yields a diffusion coefficient of $1.0 \times 10^{-7} \mathrm{~cm}^{2} / \mathrm{sec}$ and a mass transfer coefficient of $1.3 \times 10^{-\mathrm{s}} \mathrm{cm} / \mathrm{min}$. Our VLDL samples $\left(\mathrm{d}<1.006 \mathrm{~g} / \mathrm{cm}^{3}\right.$, $\mathrm{S}_{\mathrm{f}}>20$ ) most likely included some particles whose size ranged up to several thousand Angstroms (25).

Although the estimated mass transfer coefficients have a relatively large uncertainty because of neglect of concentration-dependent effects (39), they do permit semiquanitative evaluation of the degree of concentration polarization.

\section{RESULTS}

Single solutes. Fig. 4 shows the measured ultrafiltrate flux plotted as a function of applied pressure for single solutes (LDL or VLDL) in EDTA saline solution. $J_{x}$ was linearly proportional to the pressure difference, $\Delta \mathrm{P}$, when saline alone was employed. With lipoproteins

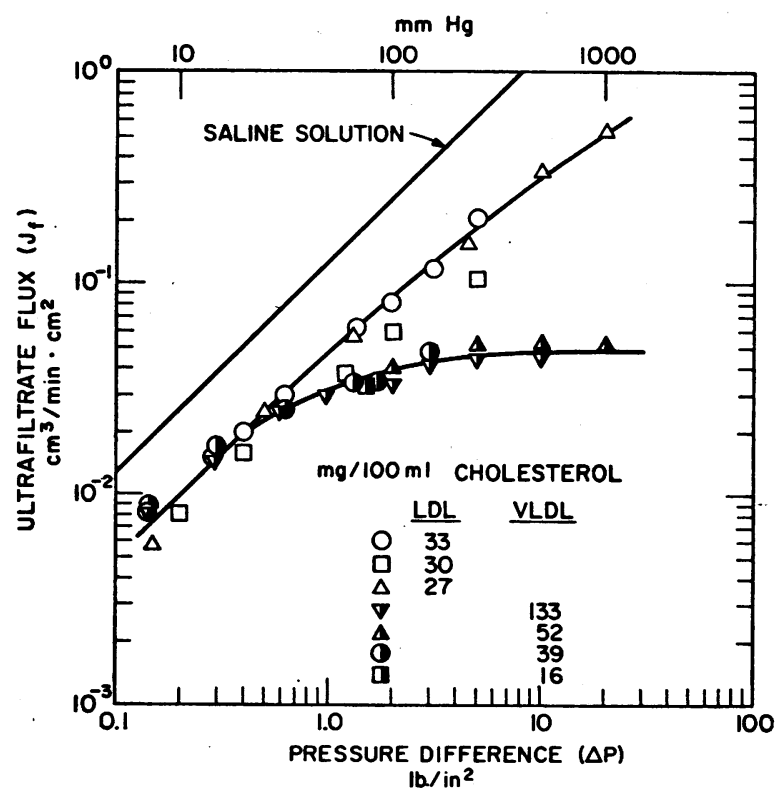

Figure 4 Dependence of ultrafiltration flux on applied pressure from measurements with solutions of single solutes. Open symbols refer to solutions of LDL and closed symbols refer to solutions of VLDL. 


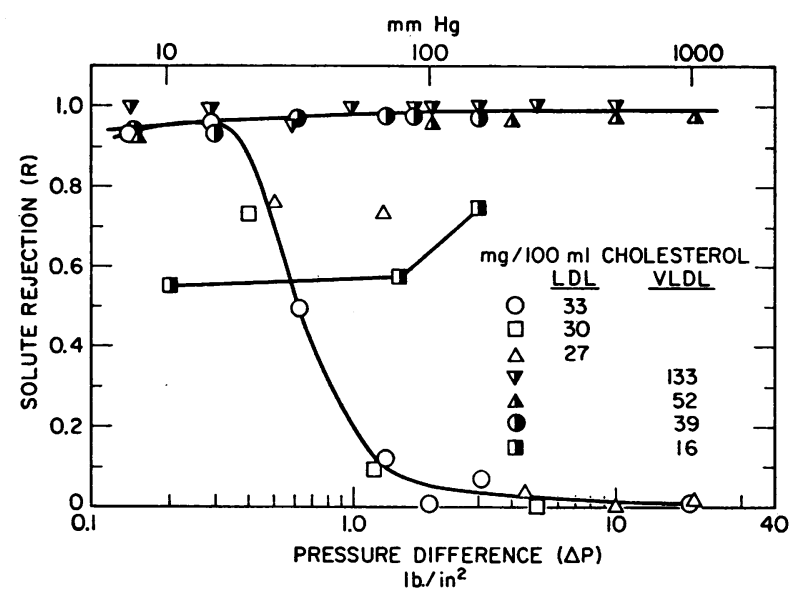

Figure 5 Dependence of observed solute (LDL or VLDL) rejection on applied pressure. Data are from the same singlesolute experiments as in Fig. 4.

present, the flux was always lower. As pressure increased, the pressure dependence for LDL solutions showed some deviation from linearity but did not reach a pressure-independent value. By contrast, the flux for solutions of VLDL showed considerable curvature and reached a constant value of about $4-5 \times 10^{-2} \mathrm{~cm}^{3}$ / $\min \cdot \mathrm{cm}^{2}$. Above about $1 \mathrm{lb}$ :/inch ${ }^{2}$, ultrafiltrate flux decreased modestly with increasing bulk concentration.

The observed solute rejection for the same single-solute experiments is shown in Fig. 5. LDL rejection increased

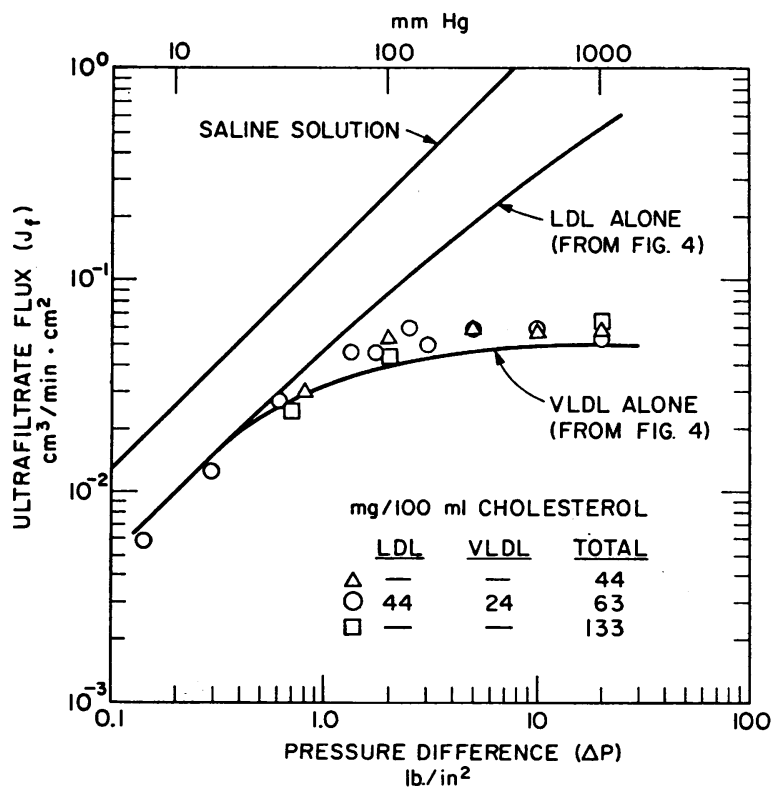

FigURE 6 Dependence of ultrafiltration flux on applied pressure with mixtures of LDL and VLDL. Solid curves correspond to smoothed mean values from single-solute experiments, Fig. 4. from 0.93 to 0.96 at low pressure, but then decreased to nearly zero as pressure and flux increased. By contrast, VLDL was nearly completely rejected at all pressures, except in dilute solution. For the two intermediate concentrations, $\mathrm{R}$ was about 0.93 at low pressures and increased to about 0.97 at high pressures. At the lowest concentration investigated $(16 \mathrm{mg} / 100 \mathrm{ml}$ cholesterol $)$, $\mathrm{R}$ was much lower. A perceptible concentration effect was also observed in the other three experiments.

Mixtures. Ultrafiltrate flux measurements with mixtures of LDL asd VLDL are compared with singlesolute data in Fig. 6. The flux in mixtures reached an asymptotic value, $5-6 \times 10^{-2} \mathrm{~cm}^{3} / \mathrm{min} \cdot \mathrm{cm}^{2}$, which was similar to the results for the more dilute solutions of pure VLDL.

The single-solute rejection data suggest that a clean separation should be possible between LDL and VLDL from mixtures of the two. However, the results for dilute mixtures (Fig. 7) showed very different behavior. At low pressures, observed VLDL rejection was low. As pressure increased, rejection rose and approached unity above about $1 \mathrm{lb}$./ inch $^{2}$, similar to the behavior of VLDL alone (Fig. 5) at the lowest concentration. More surprising, at first, was the LDL behavior in mixtures. Whereas rejection for LDL alone decreased to nearly zero with increasing pressure, in the presence of VLDL it followed qualitatively the same pattern as VLDL rejection and increased to greater than 0.9 at high pressure.

Diluted, normal, and hyperlipidemic serum. Ultrafiltrate flux is shown in Fig. 8 for a variety of solutions, including diluted serum (1:4 and $1: 1$ mixtures by volume of normal serum and buffer), normal serum, and serum from patients with type II and type IV

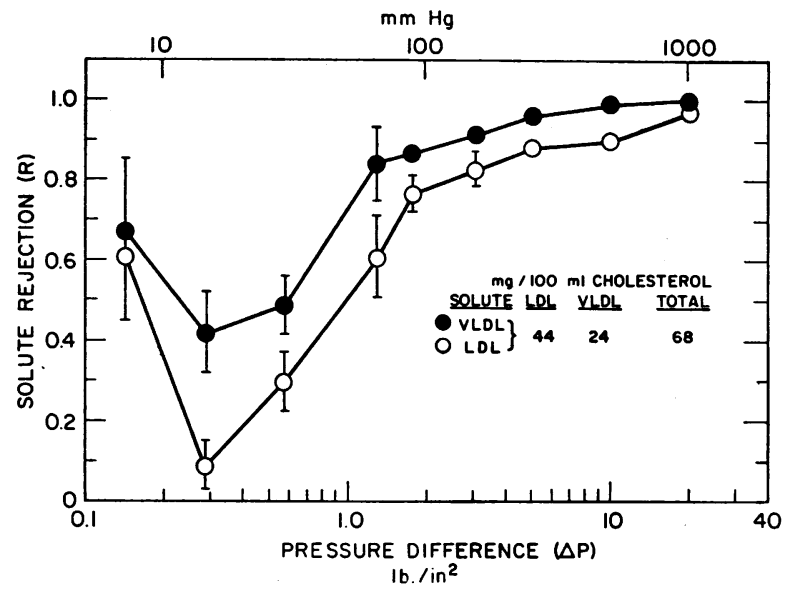

Figure 7 Effect of applied pressure on observed solute rejection of LDL and VLDL in mixtures of the two. The points shown represent the average of three runs. Error flags indicate standard deviation. 
hyperlipoproteinemia. The pathological sera were characterized by moderate elevation of LDL and normal VLDL concentration in type II and by a large increase in VLDL with normal or low LDL in type IV (40). Except for the most diluted serum, the flux reached an asymptotic value at high pressure which correlated well with VLDL concentration but not with LDL concentration.

Fig. 9 illustrates the observed LDL and VLDL rejection in normal and in diluted $(1: 4)$ serum. Within each run, VLDL rejection was higher than that for LDL. In normal serum, rejection of both solutes was high for moderate and high pressures. The results agreed well with data from mixtures (Fig. 7). At low pressures, LDL rejection was very low, less than in single-solute and LDL-VLDL mixture experiments. As pressure rose, LDL rejection increased to values approaching VLDL rejection.

Observed rejection measurements of LDL and VLDL are shown in Fig. 10 for type II and type IV serum. With type IV serum, VLDL rejection rose with increasing pressure to a limit of about 0.95. LDL followed the same pattern, approaching the VLDL rejection at high pressure. In type II serum, both LDL and VLDL showed much lower rejection and quantitatively followed a similar pattern. VLDL rejection was less

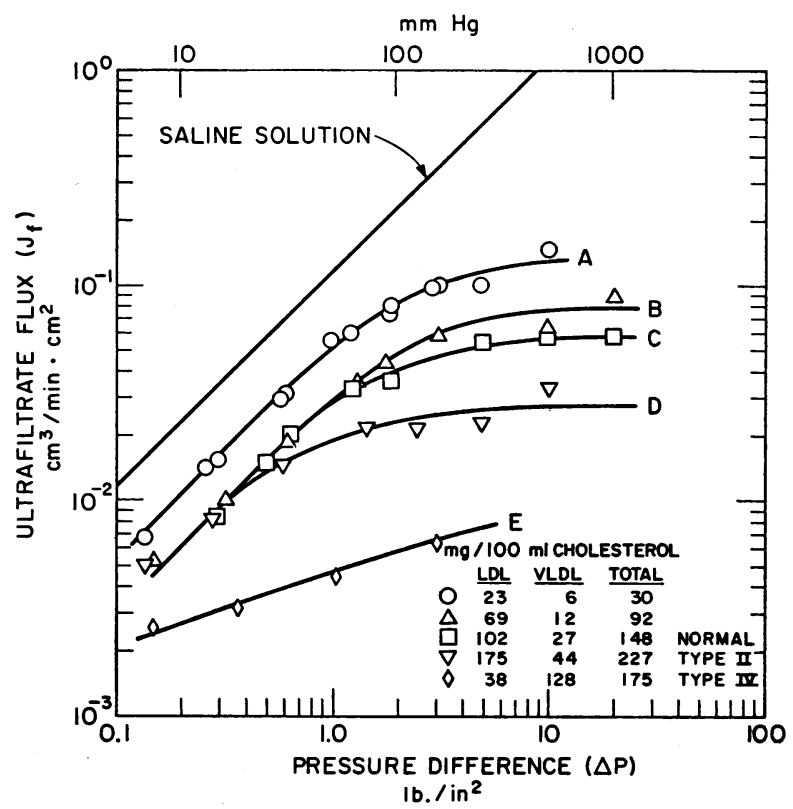

FIGURE 8 Dependence of ultrafiltration flux on applied pressure with diluted, normal, and hyperlipidemic serum. Curves are labeled as follows: A, diluted serum ( 1 part serum, 4 parts buffer); $B$, diluted serum (1 part serum, 1 part buffer); C, normal serum; $D$, serum from a patient with type II hyperlipoproteinemia; $E$, serum from a patient with type IV hyperlipoproteinemia.

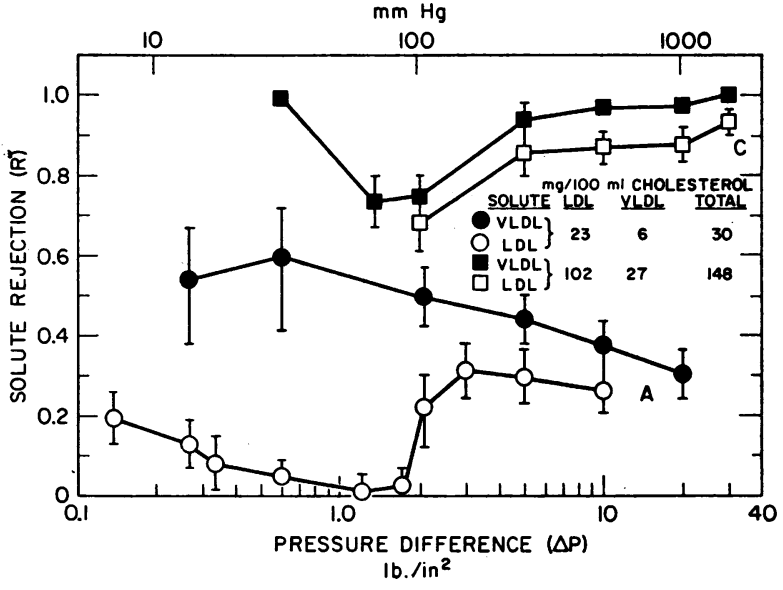

FIGURE 9 Dependence of observed solute rejection of LDL and VLDL on applied pressure in diluted and normal serum. Curves are labeled as in Fig. 8. Error flags indicate estimated standard error.

than that for LDL at all but the lowest pressures. This otherwise inexplicable observation may imply that a high concentration of LDL in some manner facilitated the transport of VLDL through the membrane, perhaps by alteration of membrane transport properties or by modification of solute behavior external to the membrane.

In Fig. 11, the LDL cholesterol flux through the membrane is plotted for type II and type IV serum. LDL cholesterol flux was 10 to 25 times greater in type II than in type IV serum. Bulk LDL cholesterol concentration in the type II serum was 4.6 times higher than in the type IV. The remaining increase was caused by the greater ultrafiltrate flux and lower LDL rejection in type II serum. VLDL cholesterol flux did not show the same magnitude of increase with type II serum as

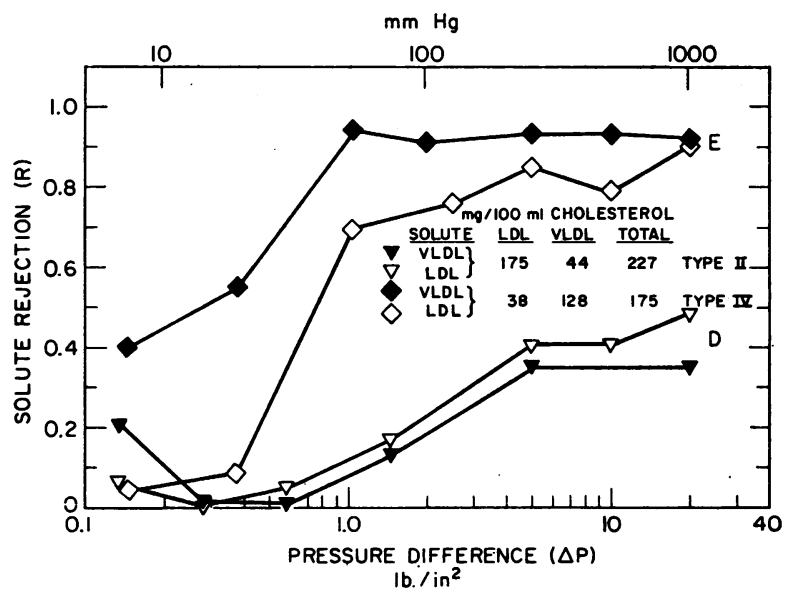

FIgURE 10 Dependence of observed solute rejection of LDL and VLDL on applied pressure in type II and type IV serum. Curves are labeled as in Fig. 8.

Ultrafiltration of Lipoproteins 


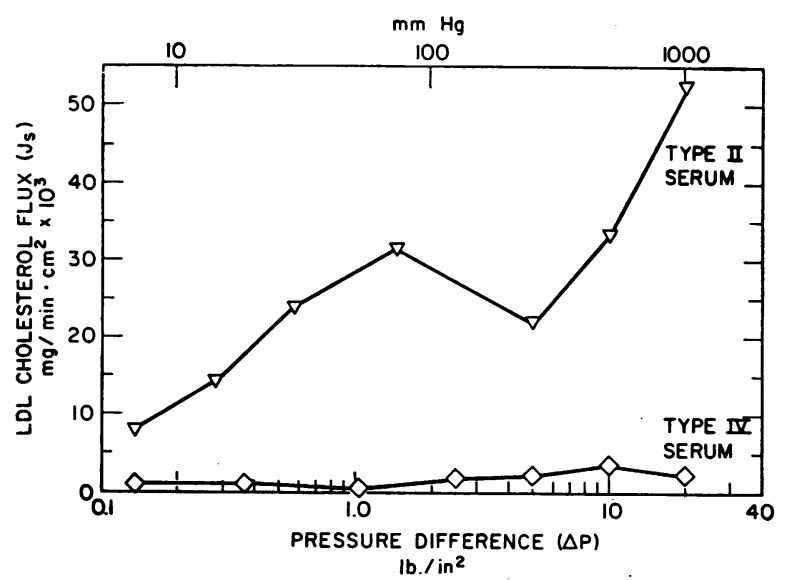

Figure 11 Dependence of LDL cholesterol flux on applied pressure in type II and type IV serum. The data are calculated from corresponding results in Figs. 8 and 10.

compared with type IV serum, because VLDL concentration in type II was much lower than in type IV.

\section{DISCUSSION}

The rate of lipoprotein transport across the synthetic membrane in these studies seems clearly to have been influenced not only by the properties of the membrane but also by the concentrated lipoprotein layer in the fluid boundary layer adjacent to the membrane. Even though this boundary layer was made turbulent by vigorous stirring, all evidence is consistent with the occurrence of considerable concentration polarization because of the very large size, and, hence, low molecular diffusivity of the lipoproteins. This phenomenon dominated the over-all transport process and obscured the intrinsic membrane properties.

The relative behavior of VLDL and LDL alone and in mixtures illustrates this effect. In solutions of pure VLDL (Fig. 4) ultrafiltrate flux reached a constant, pressure-independent value, implying that the lipoprotein had reached a maximum possible concentration $\left(c_{g}\right)$ at the membrane surface. Conversely, the continuous increase in flux with increasing pressure in solutions of pure LDL suggests that the membrane surface concentration of LDL was always less than $c_{g}$ in the concentrations studied.

In mixtures of LDL and VLDL (Fig. 6), the ultrafiltrate flux behavior was comparable with solutions of pure VLDL of similar concentration. This indicates that the maximum flux was determined largely by the solute which first reached $c_{z}$ at the membrane surface. That solute would be the largest molecule present in significant concentration. This finding was substantiated by the results obtained with serum (Fig. 8). However, the drop in flux observed between normal and type II serum was greater than would be expected on the basis of VLDL concentration alone and suggests that LDL played a significant role in flux reduction in type II serum because of its high concentration.

Intrinsic membrane rejection, $r$, may be evaluated, using equation 8 or Fig. 3 , from the observed rejection in solutions of single solutes (Fig. 5). The value of $r$ was greater than 0.95 for LDL over the entire pressure range studied. Similarly, the true membrane rejection for VLDL at the higher pressure studied was greater than 0.99 and probably greater than 0.999 .

The apparent concentration dependence of VLDL rejection shown in Figs. 5 and 9 may be explained by rewriting equation 2 :

$$
\mathrm{R}=1-\left(\frac{\mathrm{c}_{\mathrm{f}}}{\mathrm{c}_{\mathrm{w}}}\right)\left(\frac{\mathrm{c}_{\mathrm{w}}}{\mathrm{cb}_{\mathrm{b}}}\right)=1-(1-\mathrm{r}) \frac{\mathrm{c}_{\mathrm{w}}}{\mathrm{cb}_{\mathrm{b}}}
$$

If $r$ is independent of concentration and $c_{w}=c_{g}$, these two quantities may be lumped into a constant, $\mathrm{B}$, giving:

$$
\mathrm{R}=1-\frac{\mathrm{B}}{\mathrm{c}_{\mathrm{b}}}
$$

As the bulk solute concentration is varied, $R$ will vary even though $r$ is concentration independent. Since $r$ is so close to unity, the effect is significant only when $c_{b}$ is low.

The most pronounced effect in mixtures (Fig. 7) and in serum (Fig. 9 and 10) was the increase in observed LDL rejection (above that found in solutions of pure LDL) as pressure increased. The reduced ultrafiltrate flux in these solutions, which leads to a reduced polarization ratio (Fig. 2), also results in an increase in observed rejection (Fig. 3). However, this effect cannot account quantitatively for the magnitude of the increase in R. A likely explanation is that the concentrated layer of VLDL partially excludes the less polarized LDL from the region near the membrane surface. In effect, the VLDL layer acts as a second, partially rejecting membrane. This exclusion may function through a size-dependent partitioning mechanism, as in gel permeation chromatography, as well as through electrostatic repulsion since both lipoprotein classes have net negative charge. In addition, a small amount of compaction of the VLDL layer on the membrane surface with increasing pressure might increase its rejection properties. Similar phenomena have been observed with other colloidal systems $(30,41-43)$.

While concentration polarization always reduces ultrafiltrate flux, its consequences may either accelerate or reduce the transport of specific solutes. The build-up of a particular solute at the membrane surface increases its concentration at that surface and therefore increases its flux through the membrane relative to that predicted from its bulk concentration. However, with 
multiple solutes present, the component of largest molecular size may produce a second, dynamically formed membrane (in series with the primary membrane) which retards passage of the smaller solutes. The resultant of these competing influences leads to a wide variation in measured membrane properties. In our studies, LDL transport was accelerated by increased polarization and by an increase in LDL concentration, but it was reduced by a large increase in VLDL concentration. Also, VLDL transport appeared to be increased by an elevated LDL concentration. Our understanding of the mechanisms operating to produce these effects is incomplete. Nevertheless, it is clear that ultra. filtration of lipoproteins is influenced by many variables in addition to membrane properties, including ultra. filtrate flux, solute mass transfer coefficient, pressure, and the absolute and relative magnitude of LDL and VLDL concentration.

These results may have implications for the filtration theory of atherogenesis, in particular to those factors which influence the rate of lipoprotein transport from the bloodstream into the arterial wall. Theoretically, the same dimensionless parameters, $\mathrm{J} / \mathrm{k}$ and $\mathrm{r}$, which control polarization in the stirred cell also determine the degree of polarization which might occur in the arterial system, although the hydrodynamics of the two systems are markedly different. Assessment of these parameters is difficult from the sparse in vivo data which is available. Order-of-magnitude estimates, which assume rejection to be unity, have suggested that polarization of the larger lipoproteins may occur in vivo $(44,45)$.

There is reasonable evidence, however, that rejection is not unity and that these lipoproteins are transported into the arterial wall. Plasma LDL and VLDL have been shown to be present in normal and atherosclerotic arteries (46-48). The occurrence of concentration polarization within the artery would alter the rate of transport of these molecules into the wall, regardless of their subsequent fate.

Some insight into this possibility may be gained from the literature. Wilens and McCluskey studied the ultrafiltration of serum through the walls of excised arteries (13-15). During the course of their experiments, the lipid concentration inside the arteries increased markedly. This was associated with a slow decrease in ultrafiltrate flux, as would be expected if concentration polarization occurred. When the concentrated serum within the vessel was replaced by fresh serum, the flux increased to about its original level. Hence, irreversible internal plugging of the arteries was not a cause of flux reduction. They also found that ultrafiltrate concentration remained approximately constant. In other words, lipoprotein rejection increased with increasing bulk con- centration, a finding similar to our observations with the model membrane. Courtice and Garlick (12) measured the lymph-plasma concentration ratio of phospholipid and cholesterol in the leg of hypercholesterolemic rabbits before and after intravenuous infusion of an artificial fat emulsion (Lipomul, The Upjohn Co., Kalamazoo, Mich.). The observed rejection increased from about $0.5-0.7$ for phospholipid and from about 0.550.8 for cholesterol. Lipomul has an average diameter of 3000-5000 A (49). Such large particles should be subject to considerable concentration polarization at the capillary wall. The observed increase in lipid rejection parallels our observation of increased LDL rejection in the presence of VLDL.

The concentration polarization hypothesis is consistent with the focal nature of athersclerotic lesions. One would expect the local mass transfer coefficient to be lowest in regions of low shear or stagnation. At branches or bifurcations, boundary layer separation may occur on one wall, resulting in the formation of a standing vortex. This has been predicted theoretically $(50)$ and has been observed in vitro $(51,52)$ and in vivo (53). In this region, shear rates are low and stagnation occurs behind the vortex; here concentration polarization would be greatest. Boundary layer thickening without flow separation would also lead to decreased shear rates. Caro, Fitz-Gerald, and Schroter (54) have shown that the distribution of fatty streaking and early plaques is highest in these regions and that the development of lesions is inhibited in areas where the local shear rate is relatively high. They hypothesized that the occurrence of atheroma was related to a shear-dependent mass transfer process, perhaps the egress from the wall of cholesterol synthesized therein (55). As an alternative hypothesis consistent with the same observations, we propose that the movement of liproproteins from the bloodstream into the arterial wall may be the main determinant of lipid concentration in the wall and that concentration polarization is the transport-controlling mechanism.

Given the speculative nature of the existence of a polarized lipoprotein layer on the arterial intima under physiological conditions, we recognize the need for caution in extrapolating our in vitro results to in vivo conditions. It is interesting, nevertheless, to consider the effect of concentration polarization on the relationship between lipoprotein concentration in man and the rate of atherogenesis, assuming also the validity of the filtration theory of atherosclerosis. The importance of increased plasma LDL concentration in the etiology of coronary heart disease is well documented (56). Our results suggest that LDL transport into arterial wall would be favored by increased concentration polarization (for example, resulting from a locally low shear 
rate or from a high ultrafiltration flux caused by hypertension or by chemical or mechanical injury to the intimal endothelium) in conjunction with a high concentration of LDL relative to VLDL. The latter conditions are found in type II hyperlipoproteinemia (40). Similarly, decreased LDL permeation is favored by an increase in the concentration of VLDL, endogenous particles, and chylomicrons. Although these large molecules may in themselves be somewhat atherogenic, they would exert an inhibiting or protective action with regard to the smaller and more atherogenic LDL. Given the observed lipoprotein patterns in the various types of hyperlipoproteinemia, the rate of LDL transport into the arterial wall would decrease in the following order: type II $>$ IV $>$ V $>$ I. Type III is not included because its lipoprotein band having LDL electrophoretic mobility appears in the flotation fraction of normal VLDL. If this lipoprotein is assumed to be of similar size to normal LDL, then type III would be ordered between type II and type IV. It is striking, although perhaps coincidental, that this order is qualitatively consistent with the fragmentary data available concerning the occurrence of coronary atherosclerosis (57).

\section{ACKNOWLEDGMENTS}

This work was supported in part by General Clinical Research Center grant RR-88 from the Division of Research Resources, National Institutes of Health and in part by research grants from the National Heart and Lung Institute (HL 12621), the American Heart Association (69-714), and the National Dairy Council (108).

\section{REFERENCES}

1. Bader, H. 1963. The anatomy and physiology of the vascular wall. In Handbook of Physiology, Section 2: Circulation. W. F. Hamilton and P. Dow, editors. American Physiological Society, Washington, D. C. 2: 883.

2. Kellner, A. 1954. Lipid and protein content of tissue fluid in normal and hyperlipemic rabbits. Symposium on atherosclerosis. Natl. Acad. Sci. Natl. Res. Counc. Publ. No. 338, 42.

3. Evans, S. M., H. K. Ihrig, J. A. Means, W. Zeit, and E. R. Haushalter. 1952. Atherosclerosis. An in vitro study of the pathogenesis of atherosclerosis. Am. J. Clin. Pathol. 22: 354

4. Adams, C. W. M., O. B. Bayliss, and M. Z. Ibrahim. 1962. A hypothesis to explain the accumulation of cholesterol in atherosclerosis. Lancet. 1: 890.

5. Duff, G. L., and G. C. McMillan. 1951. Pathology of atherosclerosis. Am. J. Med. 11: 92 .

6. Gubner, R., and H. E. Ungerleider. 1949. Atherosclerosis: a statement of the problem. Am. J. Med. 6: 60 .

7. Page, I. H. 1954. Atherosclerosis: an introduction. Circulation. 10: 1 .

8. Wilens, S. L. 1951. The nature of diffuse intimal thickening of arteries. Am. J. Pathol. 27: 825.

9. Gofman, J. W., and W. Young. 1963. The filtration concept of atherosclerosis and serum lipids in the di- agnosis of atherosclerosis. In Atherosclerosis and Its Origin. M. Sandler and G. H. Bourne, editors. Academic Press, Inc., New York. 197.

10. Anitschkow, N. 1933. Experimental arteriosclerosis in animals. In Arteriosclerosis, a Survey of the Problem. E. V. Cowdry, editor. The Macmillan Company, New York. 298.

11. Virchow, R. 1856. Phlogose und Thrombose im GefässSystem. In Gesammelte Abhandlungen zur wissenschaftlichen Medicin. Meidinger, Sohn, Frankfurt.

12. Courtice, F. C., and D. G. Garlick. 1962. The permeability of the capillary wall to the different plasma lipoproteins of the hypercholesterolaemic rabbit in relation to their size. Quart. J. Exp. Physiol. 47: 221.

13. Wilens, S. L. 1951. The experimental production of lipid deposition in excised arteries. Science (Wash. D. C.). $114: 389$.

14. Wilens, S. L., and R. T. McCluskey. 1952. The comparative filtration properties of excised arteries and veins. Am. J. Med. Sci. 224: 540.

15. Wilens, S. L., and R. T. McCluskey. 1954. The permeability of excised arteries and other tissue to serum lipid. Circ. Res. 2 : 175.

16. Goldberg, L., and D. J. Morantz. 1957. An in-vitro study of lipid infiltration of the chick aorta. J. Pathol. Bacteriol. 74 : 1.

17. Werthessen, N. T., L. C. Milch, R. F. Redmond, L. L. Smith, and E. C. Smith. 1954. Biosynthesis and concentration of cholesterol by the intact surviving bovine aorta in vitro. Am. J. Physiol. 178: 23.

18. Duncan, L. E., Jr., and K. Buck. 1959. Passage of labeled cholesterol into the aortic wall of the normal dog. Circ. Res. $7: 765$.

19. Duncan, L. E., Jr., and K. Buck. 1960. Quantitative analysis of the development of experimental atherosclerosis in the dog. Circ. Res. 8: 1023.

20. Duncan, L. E., Jr., J. Cornfield, and K. Buck. 1962. The effect of blood pressure on the passage of labeled plasma albumin into canine aortic wall. J. Clin. Invest. $41: 1537$

21. Getz, G. S., D. Vesselinovitch, and R. W. Wissler. 1969. A dynamic pathology of atherosclerosis. Am. J. Med. 46: 657 .

22. Taylor, H. E. 1953. The role of mucopolysaccharides in the pathogenesis of intimal fibrosis and atherosclerosis of the human aorta. Am. J. Pathol. 29: 871.

23. Zugibe, F. T., and K. D. Brown. 1960. Histochemical studies in atherogenesis: human aortas. Circ. Res. 8: 287.

24. French, J. E. 1962. Atherosclerosis. In General Pathology. H. Florey, editor. W. B. Saunders, Philadelphia. 3rd edition. 418.

25. Hatch, F. T., and R. S. Lees. 1968. Practical methods for plasma lipoprotein analysis. Adv. Lipid Res. 6: 1.

26. Goldsmith, R. L. 1971. Macromolecular ultrafiltration with microporous membranes. Ind. Eng. Chem. Fundam. $10: 113$.

27. Michaels, A. S. 1968. Ultrafiltration. In Progress in Separation and Purification. E. S. Perry, editor. John Wiley \& Sons, Inc., New York. 1: 297.

28. Michaels, A. S. 1968. Separation technique for the CPI. Chem. Eng. Prog. 64: 31.

29. deFilippi, R. P., and R. L. Goldsmith. 1970. Application and theory of membrane processes for biological and other macromolecular solutions. In Membrane Science 
and Technology. J. E. Flinn, editor. Plenum Publishing Corp., New York. 33.

30. Blatt, W. F., A. Dravid, A. S. Michaels, and L. Nelsen. 1970. Solute polarization and cake formation in membrane ultrafiltration: causes, consequences, and control techniques. In Membrane Science and Technology. J. E. Fínn, editor. Plenum Publishing Corp., New York. 47.

31. Brian, P. L. T. 1966. Mass transfer in reverse osmosis. In Desalination by Reverse Osmosis. U. Merten, editor. The M.I.T. Press, Cambridge, Mass. 101.

32. Spiegler, K. S., and O. Kedem. 1966. Transport coefficients and salt rejection in uncharged hyperfiltration membranes. Desalination. $1: 311$

33. Colton, C. K. 1969. Permeability and transport studies in batch and flow dialyzers with application to hemodialysis. Ph.D. thesis. Massachusetts Institute of Technology, Cambridge, Mass.

34. Smith, K. A., C. K. Colton, E. W. Merrill, and L. B. Evans. 1968. Convective transport in a batch dialyzer: determination of true membrane permeability from a single measurement. Chem. Eng. Prog. Symp. Ser. No. 84. 64: 45.

35. Einstein, A. 1905. Über die von molekularkinetischen Theorie der Wärme geforderte Bewegung von in ruhenden Flüssigkeiten suspendierten Teilchen. Ann. Phys. (Leipzig). $17: 549$.

36. Oncley, J. L. 1964. Lipoproteins. Proceedings of the International Symposium on Lipid Transport. Nashville, 1963. H. C. Meng, Editor. Charles C. Thomas, Publisher, Springfield, Ill. 70.

37. Lindgren, F. T., A. V. Nichols, T. L. Hayes, N. K. Freeman, and J. W. Gofman. 1959. Structure and homogeneity of the low-density serum lipoproteins. Ann. N. Y. Acad. Sci. $72: 826$.

38. Jones, A. L., and J. M. Price. 1968. Some methods of electron microscopic visualization of lipoproteins in plasma and chyle. J. Histochem. Cytochem. 16: 366.

39. Keller, K. H., E. R. Canales, and S. I. Yum. 1971. Tracer and mutual diffusion coefficients of proteins. $J$. Phys. Chem. 75: 3,9.

40. Fredrickson, D. S., and R. S. Lees. 1966. Familial hyperlipoproteinemia. In The Metabolic Basis of Inherited Disease. J. B. Stanbury, J. B. Wyngaarden, and D. S. Fredrickson, editors. McGraw-Hill Book Co., New York. 2nd edition. 435.

41. van Oss, C. J., and P. M. Bronson. 1970. Separation of b'ood serum proteins by ultrafiltration. In Membrane Science and Technology. J. E. Flinn, editor. Plenum Publishing Corp., New York. 139.

42. Kraus, K. A., A. J. Shor, and J. S. Johnson, Jr. 1967. Hyperfiltration studies. X. Hyperfiltration with dynamically formed membranes. Desalination. 2: 243.

43. Kuppers, J. R., A. E. Marcinkowsky, K. A. Kraus, and J. S. Johnson. 1967. Filtration of organic solutes by dynamically formed membranes. Sep. Sci. 2: 617.
44. Keller, K. H. 1969. Mass transport phenomena in biological systems. In Biomaterials. L. Stark and G. Agarwal, editors. Plenum Publishing Corp., New York. 103.

45. Brown, C. E., M. P. Tulin, and P. van Dyke. 1970. Hydrodynamic analysis of the filtration hypothesis of lipid deposition in the genesis of arteriosclerosis. Presented at 13th Congress, Intern. Union Theoret. and Appl. Mechanics, Cambridge, Mass., 1970. (Tech. Report 611-2, Hydronautics, Inc., Laurel, Md.).

46. Hanig, M., J. R. Shainoff, and A. D. Lowy, Jr. 1956. Flotational lipoproteins extracted from human atherosclerotic aortas. Science (Wash. D. C.). 124: 176

47. Smith, E. B., and R. Slater. 1970. The chemical and immunological assay of low density lipoproteins extracted from human aortic intima. Atherosclerosis. 11: 417.

48. Scott, P. J., and P. J. Hurley. 1970. The distribution of radio-iodinated serum albumin and low density lipoprotein in tissues and the arterial wall. Atherosclerosis. $11: 77$.

49. Pinter, G. G., and D. B. Zilversmit. 1962. A gradient centrifugation method for the determination of particle size distribution of chylomicrons and of fat droplets in artificial fat emulsions. Biochim. Biophys. Acta. 59:116.

50. Hung, T. K., and S. A. Naff. 1969. A mathematical model of systolic blood flow through a bifurcation. Proceedings of the 8th International Conference on Medical and Biological Engineering and the 22nd Annual Conference on Engineering in Medicine and Biology. Chicago, Ill. 20.

51. Gutstein, W. H., and D. J. Schneck. 1967. In vitro boundary layer studies of blood flow in branched tubes. $J$. Atheroscler. Res. $7: 295$.

52. Schroter, R. C., and M. F. Sudlow. 1969. Velocity profiles in models of human airways. J. Physiol. (Lond.). 202: 36P.

53. Gutstein, W. H., G. A. Farrell, and D. J. Schneck. 1970. In vivo demonstration of junctional blood flow disturbance by hot wire anemometry. Atherosclerosis. $11: 485$.

54. Caro, C. G., J. M. Fitz-Gerald, and R. C. Schroter. 1969. Arterial wall shear and distribution of early atheroma in man. Nature (Lond.). 223: 1159.

55. Caro, C. G., J. M. Fitz-Gerald, and R. C. Schroter. 1971. Atheroma and arterial wall shear. Observation, correlation and proposal of a shear dependent mass transfer mechanism for atherogenesis. Proc. Roy. Soc. Ser. B. $177: 109$

56. Gofman, J. W., O. de Lalla, F. Glazier, N. Freeman, F. Lindgren, A. Nichols, B. Strisower, and A. Tamplin. 1954. Serum lipoprotein transport system in health, metabolic disorders, atherosclerosis and coronary artery disease. Plasma. 2: 413.

57. Fredrickson, D. S., R. I. Levy, and R. S. Lees. 1967. Fat transport in lipoproteins-an integrated approach to mechanisms and disorders. N. Engl. J. Med. 276: 34, 94, 148, 215, 273. 